\title{
Multi-parameter fourth order impulsive integral boundary value problems with one-dimensional $m$-Laplacian and deviating arguments
}

\author{
Meiqiang Feng* and Jingliang Qiu
}

\section{"Correspondence:}

meiqiangfeng@sina.com School of Applied Science, Beijing Information Science \& Technology University, Beijing, 100192, People's Republic of China

\begin{abstract}
Using inequality techniques and fixed point theories, several new and more general existence and multiplicity results are derived in terms of different values of $\lambda>0$ and $\mu>0$ for a fourth order impulsive integral boundary value problem with one-dimensional $m$-Laplacian and deviating arguments. We discuss our problems under two cases when the deviating arguments are delayed and advanced. Moreover, the nonexistence of a positive solution is also studied. In this paper, our results cover fourth order boundary value problems without deviating arguments and impulsive effect and are compared with some recent results by Jankowski.
\end{abstract}

Keywords: multi-parameter; impulsive integral boundary value problems with advanced and delayed arguments; inequality techniques and fixed point theories; one-dimensional $m$-Laplacian; existence and nonexistence of positive solutions

\section{Introduction}

Functional differential equations with impulse effect occur in many applications, such as population dynamics, biology, biotechnology, industrial robotic, pharmacokinetics, optimal control, etc., and can be expressed by functional differential equations with impulses, see [1-5]. Functional differential equations with impulses are characterized by sudden changing of their state and by the fact that the processes under consideration depend on their prehistory at each moment of time. Therefore, the study of impulsive functional differential equations has gained prominence and it is a rapidly growing field, see Zhang and Feng [6], Nieto and Rodríguez-López [7], Yan and Shen [8], Li and Shen [9], Yang and Shen [10], YS Liu [11], YJ Liu [12], He and Yu [13] and Ding et al. [14] and the references therein. We note that the difficulties dealing with such problems are that they have deviating arguments and their states are discontinuous. Therefore, the results of impulsive functional differential equations, especially for higher order impulsive functional differential equations, are fewer than those of differential equations without impulses and deviating arguments.

Moreover, owing to its importance in modeling the stationary states of the deflection of an elastic beam, fourth order boundary value problems have attracted much attention from many authors; for example, see Sun and Wang [15], Yao [16], O’Regan [17], Yang [18], 
Zhang [19], Gupta [20], Agarwal [21], Bonanno and Bella [22] and Han and Xu [23]. In particular, we would like to mention some results of Zhang and Liu [24] and Feng [25]. In [24], Zhang and Liu studied the following fourth order four-point boundary value problem without impulsive effect:

$$
\left\{\begin{array}{l}
\left(\phi_{p}\left(x^{\prime \prime}(t)\right)\right)^{\prime \prime}=w(t) f(t, x(t)), \quad t \in[0,1] \\
x(0)=0, \quad x(1)=a x(\xi), \\
x^{\prime \prime}(0)=0, \quad x^{\prime \prime}(1)=b x^{\prime \prime}(\eta)
\end{array}\right.
$$

where $0<\xi, \eta<1,0 \leq a<b<1$. By using the upper and lower solution method, fixed point theorems and the properties of Green's function $G(t, s)$ and $H(t, s)$, the authors give sufficient conditions for the existence of one positive solution.

Recently, Feng [25] studied a fourth order boundary value problem with impulses and integral boundary conditions

$$
\left\{\begin{array}{l}
\left(\phi_{p}\left(y^{\prime \prime}(t)\right)\right)^{\prime \prime}=f(t, y(t)), \quad t \in J, t \neq t_{k}, k=1,2, \ldots, n, \\
\left.\Delta y^{\prime}\right|_{t=t_{k}}=-I_{k}\left(y\left(t_{k}\right)\right), \quad k=1,2, \ldots, n, \\
y(0)=y(1)=\int_{0}^{1} g(s) y(s) d s \\
\phi_{p}\left(y^{\prime \prime}(0)\right)=\phi_{p}\left(y^{\prime \prime}(1)\right)=\int_{0}^{1} h(s) \phi_{p}\left(y^{\prime \prime}(s)\right) d s .
\end{array}\right.
$$

Using a suitably constructed cone and fixed point theory for cones, the existence of multiple positive solutions was established. Furthermore, upper and lower bounds for these positive solutions were given.

However, to the best of our knowledge, no paper has considered the existence, multiplicity and nonexistence of positive solutions for fourth order impulsive differential equations with one-dimensional $m$-Laplacian, multiple parameters and deviating arguments till now; for example, see [26-30] and the references therein.

In this paper, we investigate a fourth order impulsive integral boundary value problem with one-dimensional $m$-Laplacian and deviating arguments

$$
\left\{\begin{array}{l}
\left(\phi_{m}\left(y^{\prime \prime}(t)\right)\right)^{\prime \prime}=\lambda \omega(t) f(t, y(\alpha(t))), \quad t \in J, t \neq t_{k}, k=1,2, \ldots, n, \\
\left.\Delta y^{\prime}\right|_{t=t_{k}}=-\mu I_{k}\left(t_{k}, y\left(t_{k}\right)\right), \quad k=1,2, \ldots, n, \\
a y(0)-b y^{\prime}(0)=\int_{0}^{1} g(s) y(s) d s \\
a y(1)+b y^{\prime}(1)=\int_{0}^{1} g(s) y(s) d s \\
\phi_{p}\left(y^{\prime \prime}(0)\right)=\phi_{p}\left(y^{\prime \prime}(1)\right)=\int_{0}^{1} h(t) \phi_{p}\left(y^{\prime \prime}(t)\right) d t
\end{array}\right.
$$

where $\lambda>0$ and $\mu>0$ are two parameters, $a, b>0, J=[0,1], \phi_{m}(s)$ is an $m$-Laplace operator, i.e., $\phi_{m}(s)=|s|^{m-2} s, m>1,\left(\phi_{m}\right)^{-1}=\phi_{m^{*}}, \frac{1}{m}+\frac{1}{m^{*}}=1, t_{k}(k=1,2, \ldots, n)$ (where $n$ is a fixed positive integer) are fixed points with $0=t_{0}<t_{1}<t_{2}<\cdots<t_{k}<\cdots<t_{n}<t_{n+1}=1$, $\left.\Delta y^{\prime}\right|_{t=t_{k}}=y^{\prime}\left(t_{k}^{+}\right)-x^{\prime}\left(t_{k}^{-}\right)$, where $y^{\prime}\left(t_{k}^{+}\right)$and $y^{\prime}\left(t_{k}^{-}\right)$represent the right-hand limit and the lefthand limit of $y^{\prime}(t)$ at $t=t_{k}$, respectively. In addition, $\omega, f, I_{k}, g$ and $h$ satisfy

$\left(\mathrm{H}_{1}\right) \omega \in L^{p}[0,1]$ for some $1 \leq p \leq+\infty$, and there exists $\eta>0$ such that $\omega(t) \geq \eta$ a.e. on $J$;

$\left(\mathrm{H}_{2}\right) f: J \times R_{+} \rightarrow R_{+}$is continuous with $f(t, y)>0$ for all $t \in J$ and $y>0, \alpha \in C(J, J)$ with $R_{+}=[0,+\infty)$;

$\left(\mathrm{H}_{3}\right) I_{k}: J \times R_{+} \rightarrow R_{+}$is continuous with $I_{k}(t, y)>0(k=1,2, \ldots, n)$ for all $t$ and $y>0$; 
$\left(\mathrm{H}_{4}\right) g, h \in L^{1}[0,1]$ are nonnegative and $\xi \in[0, a), v \in[0,1)$, where

$$
\xi=\int_{0}^{1} g(t) d t, \quad v=\int_{0}^{1} h(t) d t .
$$

Some special cases of (1.1) have been investigated. For example, Jankowski [31] considered problem (1.1) with $\lambda=1, I_{k}=0$ and $\omega \in C[0,1]$, not $\omega \in L^{p}[0,1]$ for some $1 \leq p \leq+\infty$. By using a fixed point theorem for cones due to Avery and Peterson, the author proved the existence results of positive solutions for problem (1.1).

Motivated by the results mentioned above, in this paper we study the existence, multiplicity and nonexistence of positive solutions for problem (1.1) by using different methods from that of the proof of Theorem 2.1 and Theorem 2.2 in [30] to overcome difficulties arising from the appearances of $\alpha(t) \not \equiv t$ and $\omega(t)$ is $L^{p}$-integrable. The arguments are based upon a fixed point theorem due to Krasnoselskii which deals with fixed points of a cone-preserving operator defined on an ordered Banach space.

The organization of this paper is as follows. In Section 2, we present the expression and properties of Green's function associated with problem (1.1). In Section 3, we present some definitions and lemmas which are needed throughout this paper. In Section 4, we use a fixed point theorem to obtain the existence, multiplicity and nonexistence of positive solutions for problem (1.1) with advanced argument $\alpha$. In Section 5, we formulate sufficient conditions under which delayed problem (1.1) has positive solutions. In particular, our results in these sections are new when $\alpha(t) \equiv t$ on $t \in J$. Finally, in Section 6, we offer some remarks and comments of the associated problem (1.1).

\section{Expression and properties of Green's function}

We shall reduce problem (1.1) to an integral equation. To this goal, firstly by means of the transformation

$$
\phi_{m}\left(y^{\prime \prime}(t)\right)=-x(t)
$$

we convert problem (1.1) into

$$
\left\{\begin{array}{l}
x^{\prime \prime}(t)+\lambda \omega(t) f(t, y(\alpha(t)))=0, \quad t \in J \\
x(0)=x(1)=\int_{0}^{1} h(t) x(t) d t
\end{array}\right.
$$

and

$$
\left\{\begin{array}{l}
y^{\prime \prime}(t)=-\phi_{m^{*}}(x(t)), \quad t \in J, t \neq t_{k}, \\
\left.\Delta y^{\prime}\right|_{t=t_{k}}=-\mu I_{k}\left(t_{k}, y\left(t_{k}\right)\right), \quad k=1,2, \ldots, n, \\
a y(0)-b y^{\prime}(0)=\int_{0}^{1} g(s) y(s) d s, \\
a y(1)+b y^{\prime}(1)=\int_{0}^{1} g(s) y(s) d s .
\end{array}\right.
$$

Theorem 2.1 If $\left(\mathrm{H}_{1}\right),\left(\mathrm{H}_{2}\right)$ and $\left(\mathrm{H}_{4}\right)$ hold, then problem (2.2) has a unique solution $x$ given by

$$
x(t)=\lambda \int_{0}^{1} H(t, s) \omega(s) f(s, y(\alpha(s))) d s,
$$


where

$$
\begin{aligned}
& H(t, s)=G(t, s)+\frac{1}{1-v} \int_{0}^{1} G(s, \tau) h(\tau) d \tau, \\
& G(t, s)= \begin{cases}t(1-s), & 0 \leq t \leq s \leq 1 \\
s(1-t), & 0 \leq s \leq t \leq 1 .\end{cases}
\end{aligned}
$$

Proof The proof of Theorem 2.1 is similar to that of Lemma 2.1 in [25].

Write $e(t)=t(1-t)$. Then from (2.5) and (2.6) we can prove that $H(t, s)$ and $G(t, s)$ have the following properties.

Theorem 2.2 Let $G$ and $H$ be given as in Theorem 2.1. If $\left(\mathrm{H}_{4}\right)$ holds, then

$$
\begin{aligned}
& H(t, s)>0, \quad G(t, s)>0, \quad \forall t, s \in(0,1), \\
& H(t, s) \geq 0, \quad G(t, s) \geq 0, \quad \forall t, s \in J, \\
& e(t) e(s) \leq G(t, s) \leq G(t, t)=t(1-t)=e(t) \leq \bar{e}=\max _{t \in J} e(t)=\frac{1}{4}, \quad \forall t, s \in J, \\
& \rho e(s) \leq H(t, s) \leq \gamma s(1-s)=\gamma e(s) \leq \frac{1}{4} \gamma, \quad \forall t, s \in J,
\end{aligned}
$$

where

$$
\gamma=\frac{1}{1-v}, \quad \rho=\frac{\int_{0}^{1} e(\tau) h(\tau) d \tau}{1-v} .
$$

Theorem 2.3 If $\left(\mathrm{H}_{1}\right),\left(\mathrm{H}_{3}\right)$ and $\left(\mathrm{H}_{4}\right)$ hold, then problem (2.3) has a unique solution y expressed in the form

$$
y(t)=\int_{0}^{1} H_{1}(t, s) \phi_{m^{*}}(x(s)) d s+\mu \sum_{k=1}^{n} H_{1}\left(t, t_{k}\right) I_{k}\left(t_{k}, y\left(t_{k}\right)\right),
$$

where

$$
\begin{aligned}
& H_{1}(t, s)=G_{1}(t, s)+\frac{1}{a-\xi} \int_{0}^{1} G_{1}(s, \tau) g(\tau) d \tau \\
& G_{1}(t, s)=\frac{1}{d} \begin{cases}(b+a s)(b+a(1-t)), & \text { if } 0 \leq s \leq t \leq 1, \\
(b+a t)(b+a(1-s)), & \text { if } 0 \leq t \leq s \leq 1\end{cases}
\end{aligned}
$$

and

$$
d=a(2 b+a)
$$

Proof The proof of Theorem 2.3 is similar to that of Lemma 2.2 in [25].

From (2.13) and (2.14) we can prove that $H_{1}(t, s)$ and $G_{1}(t, s)$ have the following properties. 
Theorem 2.4 Let $\zeta \in\left(0, t_{1}\right), G_{1}$ and $H_{1}$ be given as in Theorem 2.3. If $\left(\mathrm{H}_{4}\right)$ holds, then we have

$$
\begin{aligned}
& \frac{1}{d} b^{2} \leq G_{1}(t, s) \leq G_{1}(s, s) \leq \frac{1}{d}(b+a)^{2}, \quad \forall t, s \in J, \\
& \rho_{1} \leq H_{1}(t, s) \leq \frac{a}{a-\xi} G_{1}(s, s) \leq \rho_{2}, \quad \forall t, s \in J, \\
& G_{1}(t, s) \geq \delta G_{1}(s, s) \geq \frac{b^{2} \delta}{d}, \quad H_{1}(t, s) \geq \frac{\delta a}{a-\xi} G_{1}(s, s) \geq \delta \rho_{1}, \quad \forall t \in[\zeta, 1], s \in J,
\end{aligned}
$$

where

$$
\delta=\frac{b}{a+b}, \quad \rho_{1}=\frac{b^{2} \gamma_{1}}{a+2 b}, \quad \rho_{2}=\frac{\gamma_{1}(b+a)^{2}}{a+2 b}, \quad \gamma_{1}=\frac{1}{a-\xi} .
$$

Proof It follows from the definition of $G_{1}(t, s)$ and $H_{1}(t, s)$ that (2.15) and (2.16) hold. Now, we show that (2.17) also holds.

In fact, for $t \in[\zeta, 1]$ and $s \in J$, we have that

$$
\begin{aligned}
& \frac{G_{1}(t, s)}{G_{1}(s, s)}=\frac{b+a(1-t)}{b+a(1-s)} \geq \frac{b}{b+a} \quad \text { for } s \leq t, \\
& \frac{G_{1}(t, s)}{G_{1}(s, s)}=\frac{b+a t}{b+a s} \geq \frac{b+a \zeta}{b+a} \quad \text { for } t \leq s .
\end{aligned}
$$

This and (2.13) show that

$$
\begin{aligned}
H_{1}(t, s) & \geq \delta G_{1}(s, s)\left[1+\frac{1}{a-\xi} \int_{0}^{1} g(\tau) d \tau\right] \\
& \geq \frac{a \delta}{a-\xi} G_{1}(s, s), \quad t \in[\zeta, 1], s \in J .
\end{aligned}
$$

This together with (2.13) and (2.15) finishes the proof of (2.17).

From Theorem 2.1 and Theorem 2.3, we have the following result.

Theorem 2.5 Assume that $\left(\mathrm{H}_{1}\right)-\left(\mathrm{H}_{4}\right)$ hold. Then problem (1.1) has a unique solution $y$ given by

$$
\begin{aligned}
y(t)= & \int_{0}^{1} H_{1}(t, s) \phi_{m^{*}}\left(\lambda \int_{0}^{1} H(s, \tau) \omega(\tau) f(\tau, y(\alpha(\tau))) d \tau\right) d s \\
& +\mu \sum_{k=1}^{n} H_{1}\left(t, t_{k}\right) I_{k}\left(t_{k}, y\left(t_{k}\right)\right) .
\end{aligned}
$$

\section{Preliminaries for the case $\alpha(t) \geq t$ on $J$}

We begin by introducing the notations

$$
\begin{aligned}
& f^{0}=\limsup _{y \rightarrow 0^{+}} \max _{t \in J} \frac{f(t, y)}{\phi_{m}(y)}, \quad f^{\infty}=\limsup _{y \rightarrow \infty} \max _{t \in J} \frac{f(t, y)}{\phi_{m}(y)}, \\
& f_{0}=\liminf _{y \rightarrow 0^{+}} \min _{t \in J} \frac{f(t, y)}{\phi_{m}(y)}, \quad f_{\infty}=\liminf _{y \rightarrow \infty} \min _{t \in J} \frac{f(t, y)}{\phi_{m}(y)},
\end{aligned}
$$




$$
\begin{array}{ll}
I^{0}(k)=\limsup _{y \rightarrow 0^{+}} \max _{t \in J} \frac{I_{k}(t, y)}{y}, & I^{\infty}(k)=\limsup _{y \rightarrow \infty} \max _{t \in J} \frac{I_{k}(t, y)}{y}, \\
I_{0}(k)=\liminf _{y \rightarrow 0^{+}} \min _{t \in J} \frac{I_{k}(t, y)}{y}, & I_{\infty}(k)=\liminf _{y \rightarrow \infty} \min _{t \in J} \frac{I_{k}(t, y)}{y}, \quad k=1,2, \ldots, n .
\end{array}
$$

We will also need the functions

$$
f^{*}(u)=\max \left\{\max _{t \in J} f(t, y), y \in[0, u]\right\}, \quad I_{k}^{*}(u)=\max \left\{\max _{t \in J} I_{k}(t, y), y \in[0, u]\right\}
$$

and let

$$
\begin{array}{ll}
f_{0}^{*}=\lim _{u \rightarrow 0^{+}} \frac{f^{*}(u)}{\phi_{m}(u)}, & f_{\infty}^{*}=\lim _{u \rightarrow \infty} \frac{f^{*}(u)}{\phi_{m}(u)}, \\
I_{0}^{*}(k)=\lim _{u \rightarrow 0^{+}} \frac{I_{k}^{*}(u)}{u}, & I_{\infty}^{*}(k)=\lim _{u \rightarrow \infty} \frac{I_{k}^{*}(u)}{u},
\end{array}
$$

where $k=1,2, \ldots, n$.

Our first lemma gives some relationships between the functions $f$ and $f^{*}$ and $I_{k}^{*}$ and $I_{k}$.

Lemma 3.1 (See [32]) Assume that $\left(\mathrm{H}_{1}\right)$ holds. Then

$$
f_{0}^{*}=f^{0}, \quad f_{\infty}^{*}=f^{\infty}, \quad I_{0}^{*}(k)=I^{0}(k), \quad I_{\infty}^{*}(k)=I^{\infty}(k),
$$

where $k=1,2, \ldots, n$.

Proof The proof of Lemma 3.1 is similar to that of Lemma 2.8 in [32].

The following fixed point theorem of a cone is crucial in the proofs of our results.

Lemma 3.2 (See [33]) Let $P$ be a cone in a real Banach space E. Assume that $\Omega_{1}$ and $\Omega_{2}$ are bounded open sets in $E$ with $0 \in \Omega_{1}, \bar{\Omega}_{1} \subset \Omega_{2}$. If

$$
A: P \cap\left(\bar{\Omega}_{2} \backslash \Omega_{1}\right) \rightarrow P
$$

is completely continuous such that either

(i) $\|A x\| \leq\|x\|, \forall x \in P \cap \partial \Omega_{1}$ and $\|A x\| \geq\|x\|, \forall x \in P \cap \partial \Omega_{2}$, or

(ii) $\|A x\| \geq\|x\|, \forall x \in P \cap \partial \Omega_{1}$ and $\|A x\| \leq\|x\|, \forall x \in P \cap \partial \Omega_{2}$, then $A$ has at least one fixed point in $P \cap\left(\bar{\Omega}_{2} \backslash \Omega_{1}\right)$.

Let $J^{\prime}=J \backslash\left\{t_{1}, t_{2}, \ldots, t_{m}\right\}$, and

$$
P C^{1}[0,1]=\left\{y \in C[0,1]:\left.y^{\prime}\right|_{\left(t_{k}, t_{k+1}\right)} \in C\left(t_{k}, t_{k+1}\right), y^{\prime}\left(t_{k}^{-}\right), y^{\prime}\left(t_{k}^{+}\right) \text {exist, } k=1,2, \ldots, m\right\} .
$$

Then $P C^{1}[0,1]$ is a real Banach space with the norm

$$
\|y\|_{P C^{1}}=\max \left\{\|y\|_{\infty},\left\|y^{\prime}\right\|_{\infty}\right\}
$$

where $\|y\|_{\infty}=\sup _{t \in J}|y(t)|,\left\|y^{\prime}\right\|_{\infty}=\sup _{t \in J}\left|y^{\prime}(t)\right|$. 
A function $y \in P C^{1}[0,1] \cap C^{4}\left(J^{\prime}\right)$ with $\varphi_{p}\left(y^{\prime \prime}\right) \in C^{2}(0,1)$ is called a solution of problem (1.1) if it satisfies (1.1).

Define a cone in $P C^{1}[0,1]$ by

$$
K=\left\{y \in P C^{1}[0,1]: y(t) \geq 0 \text { on } J \text { and } \min _{t \in[\zeta, 1]} y(t) \geq \delta \frac{\rho_{1}}{\rho_{4}}\|y\|_{P C^{1}}\right\}
$$

where $\delta, \rho_{1}$ and $\rho_{4}$ are defined in (2.18) and (3.8), respectively. For $r>0$, let

$$
\Omega_{r}=\left\{y \in K:\|y\|_{P C^{1}}<r\right\} .
$$

Define an operator $T_{\lambda}^{\mu}: K \rightarrow P C^{1}[0,1]$ by

$$
\begin{aligned}
\left(T_{\lambda}^{\mu} y\right)(t)= & \int_{0}^{1} H_{1}(t, s) \phi_{m^{*}}\left(\lambda \int_{0}^{1} H(s, \tau) \omega(\tau) f(\tau, y(\alpha(\tau))) d \tau\right) d s \\
& +\mu \sum_{k=1}^{n} H_{1}\left(t, t_{k}\right) I_{k}\left(t_{k}, y\left(t_{k}\right)\right) .
\end{aligned}
$$

It follows from (3.4) and Theorem 2.5 that the following lemma holds.

Lemma 3.3 Assume that $\left(\mathrm{H}_{1}\right)-\left(\mathrm{H}_{4}\right)$ hold. Then $y \in K$ is a positive fixed point of $T_{\lambda}^{\mu}$ if and only ify is a positive solution of problem (1.1).

Lemma 3.4 Suppose that $\left(\mathrm{H}_{1}\right)-\left(\mathrm{H}_{4}\right)$ hold. Then $T_{\lambda}^{\mu}(K) \subset K$ and $T_{\lambda}^{\mu}: K \rightarrow K$ is completely continuous.

Proof For all $u \in K$, then $T_{\lambda} u \geq 0$ on $J$ and it follows from (2.16) and (3.4) that

$$
\begin{aligned}
\left(T_{\lambda}^{\mu} y\right)(t)= & \int_{0}^{1} H_{1}(t, s) \phi_{m^{*}}\left(\lambda \int_{0}^{1} H(s, \tau) \omega(\tau) f(\tau, y(\alpha(\tau))) d \tau\right) d s \\
& +\mu \sum_{k=1}^{n} H_{1}\left(t, t_{k}\right) I_{k}\left(t_{k}, y\left(t_{k}\right)\right) \\
\leq & \rho_{2}\left(\int_{0}^{1} \phi_{m^{*}}\left(\lambda \int_{0}^{1} H(s, \tau) \omega(\tau) f(\tau, y(\alpha(\tau))) d \tau\right) d s+\mu \sum_{k=1}^{n} I_{k}\left(t_{k}, y\left(t_{k}\right)\right)\right) \\
& t \in J \\
\left|\left(T_{\lambda}^{\mu} y\right)^{\prime}(t)\right| \leq & \int_{0}^{1}\left|H_{1 t}^{\prime}(t, s)\right| \phi_{m^{*}}\left(\lambda \int_{0}^{1} H(s, \tau) \omega(\tau) f(\tau, y(\tau)) d \tau\right) d s \\
& +\mu \sum_{k=1}^{m}\left|H_{1 t}^{\prime}\left(t, t_{k}\right)\right| I_{k}\left(t_{k}, y\left(t_{k}\right)\right) \\
\leq & \rho_{3}\left(\int_{0}^{1} \phi_{m^{*}}\left(\lambda \int_{0}^{1} H(s, \tau) \omega(\tau) f(\tau, y(\alpha(\tau))) d \tau\right) d s+\mu \sum_{k=1}^{n} I_{k}\left(t_{k}, y\left(t_{k}\right)\right)\right) \\
& t \in J,
\end{aligned}
$$


where $\rho_{3}=\frac{1}{d} a(a+b)$,

$$
H_{1 t}^{\prime}(t, s)=G_{1 t}^{\prime}(t, s)=\frac{1}{d} \begin{cases}-a(b+a s), & \text { if } 0 \leq s \leq t \leq 1 \\ a(b+a(1-s)), & \text { if } 0 \leq t \leq s \leq 1\end{cases}
$$

and

$$
\max _{t, s \in J, t \neq s}\left|H_{1 t}^{\prime}(t, s)\right|=\max _{t, s \in J, t \neq s}\left|G_{1 t}^{\prime}(t, s)\right|=\frac{1}{d} a(b+a) .
$$

It follows from (3.5) and (3.6) that

$$
\begin{aligned}
\left\|T_{\lambda}^{\mu} y\right\|_{P C^{1}} \leq & \rho_{4}\left(\int_{0}^{1} \phi_{m^{*}}\left(\lambda \int_{0}^{1} H(s, \tau) \omega(\tau) f(\tau, y(\alpha(\tau))) d \tau\right) d s\right. \\
& \left.+\mu \sum_{k=1}^{n} I_{k}\left(t_{k}, y\left(t_{k}\right)\right)\right)
\end{aligned}
$$

where

$$
\rho_{4}=\max \left\{\rho_{2}, \rho_{3}\right\}
$$

Noticing (2.17), (3.4) and (3.7), we have

$$
\begin{aligned}
\min _{t \in[\zeta, 1]}\left(T_{\lambda}^{\mu} y\right)(t) \\
=\min _{t \in[\zeta, 1]}\left(\int_{0}^{1} H_{1}(t, s) \phi_{m^{*}}\left(\lambda \int_{0}^{1} H(s, \tau) \omega(\tau) f(\tau, y(\alpha(\tau))) d \tau\right) d s\right. \\
\left.\quad+\mu \sum_{k=1}^{n} H_{1}\left(t, t_{k}\right) I_{k}\left(t_{k}, y\left(t_{k}\right)\right)\right) \\
\geq \delta \rho_{1}\left(\int_{0}^{1} \phi_{m^{*}}\left(\lambda \int_{0}^{1} H(s, \tau) \omega(\tau) f(\tau, y(\alpha(\tau))) d \tau\right) d s+\mu \sum_{k=1}^{n} I_{k}\left(t_{k}, y\left(t_{k}\right)\right)\right) \\
=\delta \frac{\rho_{1}}{\rho_{4}} \rho_{4}\left(\int_{0}^{1} \phi_{m^{*}}\left(\lambda \int_{0}^{1} H(s, \tau) \omega(\tau) f(\tau, y(\alpha(\tau))) d \tau\right) d s+\mu \sum_{k=1}^{n} I_{k}\left(t_{k}, y\left(t_{k}\right)\right)\right) \\
\geq \delta \frac{\rho_{1}}{\rho_{4}}\left\|T_{\lambda}^{\mu} y\right\|_{P C^{1}} .
\end{aligned}
$$

Thus, $T_{\lambda}^{\mu}(K) \subset K$.

Finally, similar to the proof of Lemma 2.10 in [25], one can prove that $T_{\lambda}^{\mu}: K \rightarrow K$ is completely continuous. This gives the proof of Lemma 3.4.

To obtain some of the norm inequalities in Lemma 3.6 and Lemma 3.8, we employ Hölder's inequality.

Lemma 3.5 (Hölder) Let $f \in L^{p}[a, b]$ with $p>1, g \in L^{q}[a, b]$ with $q>1$, and $\frac{1}{p}+\frac{1}{q}=1$. Then $f g \in L^{1}[a, b]$ and

$$
\|f g\|_{1} \leq\|f\|_{p}\|g\|_{q}
$$


Let $f \in L^{1}[a, b], g \in L^{\infty}[a, b]$. Then $f g \in L^{1}[a, b]$ and

$\|f g\|_{1} \leq\|f\|_{1}\|g\|_{\infty}$.

Next, we consider the following cases for $\omega \in L^{p}[0,1]: p>1, p=1, p=\infty$. Case $p>1$ is treated in Lemma 3.6 and Lemma 3.8.

Lemma 3.6 Assume that $\left(\mathrm{H}_{1}\right)-\left(\mathrm{H}_{4}\right)$ hold, $\alpha(t) \geq t$ on $J$ and let $r>0$ be given. If there exist $\varepsilon_{1}>0$ and $\varepsilon_{2}>0$ such that $f^{*}(r) \leq \varepsilon_{1} \phi_{m}(r)$ and $I_{k}^{*}(r) \leq \varepsilon_{2} r(k=1,2, \ldots, n)$, then

$$
\left\|T_{\lambda}^{\mu} y\right\|_{P C^{1}} \leq \rho_{4}\left(\phi_{m^{*}}\left(\lambda \gamma \varepsilon_{1}\|e\|_{q}\|\omega\|_{p}\right)+\mu n \varepsilon_{2}\right)\|y\|_{P C^{1}}, \quad y \in \partial \Omega_{r} .
$$

Proof By the definition of $f^{*}(r)$ and $I_{k}^{*}$, if $f^{*}(r) \leq \varepsilon_{1} \phi_{m}(r)$ and $I_{k}^{*}(r) \leq \varepsilon_{2} r(k=1,2, \ldots, n)$, then

$$
f(t, y) \leq \varepsilon_{1} \phi_{m}(r), \quad I_{k}(t, y) \leq \varepsilon_{2} r \quad \text { for } t \in J \text { and } 0 \leq y \leq r .
$$

Since $0 \leq t \leq \alpha(t) \leq 1$ on $J$, it follows from $0 \leq y(t) \leq r$ on $J$ that $0 \leq y(\alpha(t)) \leq r$.

Therefore, we have $f(t, y(\alpha(t))) \leq \varepsilon_{1} \phi_{m}(r)$ for $t \in J$, and it follows from (3.7) that

$$
\begin{aligned}
\left\|T_{\lambda}^{\mu} y\right\|_{P C^{1}} & \leq \rho_{4}\left(\int_{0}^{1} \phi_{m^{*}}\left(\lambda \int_{0}^{1} H(s, \tau) \omega(\tau) f(\tau, y(\alpha(\tau))) d \tau\right) d s+\mu \sum_{k=1}^{n} I_{k}\left(t_{k}, y\left(t_{k}\right)\right)\right) \\
& \leq \rho_{4}\left(\phi_{m^{*}}\left(\lambda \int_{0}^{1} \gamma e(\tau) \omega(\tau) f(\tau, y(\alpha(\tau))) d \tau\right)+\mu \sum_{k=1}^{n} I_{k}\left(t_{k}, y\left(t_{k}\right)\right)\right) \\
& \leq \rho_{4}\left(\phi_{m^{*}}\left(\lambda \gamma \int_{0}^{1} e(\tau) \omega(\tau) \varepsilon_{1} \phi_{m}(r) d \tau\right)+\mu \sum_{k=1}^{n} \varepsilon_{2} r\right) \\
& =\rho_{4}\left(\phi_{m^{*}}\left(\lambda \gamma \varepsilon_{1} \phi_{m}(r) \int_{0}^{1} e(\tau) \omega(\tau) d \tau\right)+\mu \sum_{k=1}^{n} \varepsilon_{2} r\right) \\
& \leq \rho_{4}\left(\phi_{m^{*}}\left(\lambda \gamma \varepsilon_{1} \phi_{m}(r)\|e\|_{q}\|\omega\|_{p}\right)+\mu n \varepsilon_{2} r\right) \\
& =\rho_{4}\left(\phi_{m^{*}}\left(\lambda \gamma \varepsilon_{1}\|e\|_{q}\|\omega\|_{p}\right)+\mu n \varepsilon_{2} r\right)\|y\|_{P C^{1}}, \quad \forall y \in \partial \Omega_{r} .
\end{aligned}
$$

This completes the proof.

The following result deals with the case $p=1$.

Corollary 3.1 Assume that $\left(\mathrm{H}_{1}\right)-\left(\mathrm{H}_{4}\right)$ hold, $\alpha(t) \geq t$ on $J$ and let $r>0$ be given. If there exist $\varepsilon_{1}>0$ and $\varepsilon_{2}>0$ such that $f^{*}(r) \leq \varepsilon_{1} \phi_{m}(r)$ and $I_{k}^{*}(r) \leq \varepsilon_{2} r(k=1,2, \ldots, n)$, then

$$
\left\|T_{\lambda}^{\mu} y\right\|_{P C^{1}} \leq \rho_{4}\left(\phi_{m^{*}}\left(\lambda \gamma \varepsilon_{1}\|e\|_{\infty}\|\omega\|_{1}\right)+\mu n \varepsilon_{2} r\right)\|y\|_{P C^{1}}, \quad y \in \partial \Omega_{r} .
$$

Proof By Lemma 3.6, let $\|e\|_{\infty}\|\omega\|_{1}$ replace $\|e\|_{q}\|\omega\|_{p}$ and repeat the argument above.

Finally we consider the case of $p=\infty$. 
Corollary 3.2 Assume that $\left(\mathrm{H}_{1}\right)-\left(\mathrm{H}_{4}\right)$ hold, $\alpha(t) \geq t$ on $J$ and let $r>0$ be given. If there exist $\varepsilon_{1}>0$ and $\varepsilon_{2}>0$ such that $f^{*}(r) \leq \varepsilon_{1} \phi_{m}(r)$ and $I_{k}^{*}(r) \leq \varepsilon_{2} r(k=1,2, \ldots, n)$, then

$$
\left\|T_{\lambda}^{\mu} y\right\|_{P C^{1}} \leq \rho_{4}\left(\phi_{m^{*}}\left(\lambda \gamma \varepsilon_{1}\|e\|_{1}\|\omega\|_{\infty}\right)+\mu n \varepsilon_{2} r\right)\|y\|_{P C^{1}}, \quad y \in \partial \Omega_{r} .
$$

Proof By Lemma 3.6, let $\|e\|_{1}\|\omega\|_{\infty}$ replace $\|e\|_{q}\|\omega\|_{p}$ and repeat the argument above.

Lemma 3.7 Assume that $\left(\mathrm{H}_{1}\right)-\left(\mathrm{H}_{4}\right)$ hold, $\alpha(t) \geq t$ on $J$ and let $l_{1}>0$ and $l_{2}$ be given. If $f(t, y) \geq l_{1} \phi_{m}(y)$ and $I_{k}(t, y) \geq l_{2} y(k=1,2, \ldots, n)$ for $t \in J$ and $y \in K$, then

$$
\left\|T_{\lambda}^{\mu} y\right\|_{P C^{1}} \geq \delta \frac{\rho_{1}^{2}}{\rho_{4}}\left(\left(l_{1} \lambda \rho \eta\right)^{m^{*}-1} \beta+\mu n l_{2}\right)\|y\|_{P C^{1}},
$$

where $\beta=\phi_{m^{*}}\left(\int_{\zeta}^{1} e(\tau) d \tau\right)$.

Proof By the definition of $K$, if $y \in K$, then we have

$$
y(t) \geq 0 \quad \text { on } J \quad \text { and } \quad \min _{t \in[\zeta, 1]} y(t) \geq \delta \frac{\rho_{1}}{\rho_{4}}\|y\|_{P C^{1}} .
$$

Since $0 \leq t \leq \alpha(t) \leq 1$ on $J$, it follows from $y(t) \geq 0$ on $J$ that $y(\alpha(t)) \geq 0$.

Similarly, since $\zeta \leq t \leq \alpha(t) \leq 1$ on $[\zeta, 1]$, it follows from $\min _{t \in[\zeta, 1]} y(t) \geq \delta \frac{\rho_{1}}{\rho_{4}}\|y\|_{P C^{1}}$ that

$$
\min _{t \in[\zeta, 1]} y(\alpha(t)) \geq \delta \frac{\rho_{1}}{\rho_{4}}\|y\|_{P C^{1}}
$$

Therefore, $f(t, y(\alpha(t))) \geq l_{1} \phi_{m}(y)$ for $t \in J$, and it follows from the definition of $T_{\lambda}^{\mu}$ and (2.17) that

$$
\begin{aligned}
\left\|T_{\lambda}^{\mu} y\right\|_{P C^{1}} & \geq \rho_{1} \phi_{m^{*}}\left(\lambda \int_{0}^{1} \rho e(\tau) \omega(\tau) f(\tau, y(\alpha(\tau))) d \tau\right)+\mu \rho_{1} \sum_{k=1}^{n} I_{k}\left(t_{k}, y\left(t_{k}\right)\right) \\
& \geq \rho_{1}(\lambda \rho \eta)^{m^{*}-1} \phi_{m^{*}}\left(\int_{0}^{1} e(\tau) f(\tau, y(\alpha(\tau))) d \tau\right)+\mu \rho_{1} \sum_{k=1}^{n} I_{k}\left(t_{k}, y\left(t_{k}\right)\right) \\
& \geq \rho_{1}(\lambda \rho \eta)^{m^{*}-1} \phi_{m^{*}}\left(\int_{0}^{1} e(\tau) l_{1} \phi_{m}(y(\alpha(\tau))) d \tau\right)+\mu \rho_{1} \sum_{k=1}^{n} l_{2} y\left(t_{k}\right) \\
& \geq \rho_{1}\left(l_{1} \lambda \rho \eta\right)^{m^{*}-1} \phi_{m^{*}}\left(\int_{\zeta}^{1} e(\tau) \phi_{m}(y(\alpha(\tau))) d \tau\right)+\mu \rho_{1} l_{2} \sum_{k=1}^{n} y\left(t_{k}\right) \\
& \geq \rho_{1}\left(l_{1} \lambda \rho \eta\right)^{m^{*}-1} \phi_{m^{*}}\left(\int_{\zeta}^{1} e(\tau) \phi_{m}\left(\delta \frac{\rho_{1}}{\rho_{4}}\|y\|_{P C^{1}}\right) d \tau\right)+\mu \rho_{1} l_{2} \sum_{k=1}^{n} \delta \frac{\rho_{1}}{\rho_{4}}\|y\|_{P C^{1}} \\
& =\delta \frac{\rho_{1}^{2}}{\rho_{4}}\left(\left(l_{1} \lambda \rho \eta\right)^{m^{*}-1} \beta+\mu n l_{2}\right)\|y\|_{P C^{1}} .
\end{aligned}
$$

This completes the proof.

Lemma 3.8 Assume that $\left(\mathrm{H}_{1}\right)-\left(\mathrm{H}_{4}\right)$ hold and $\alpha(t) \geq t$ on J. If $y \in \partial \Omega_{r}, r>0$, then

$$
\left\|T_{\lambda}^{\mu} y\right\|_{P C^{1}} \leq \rho_{4}\left(\phi_{m^{*}}\left(\lambda \gamma M_{r}\|e\|_{q}\|\omega\|_{p}\right)+\mu n M^{*}\right)
$$


where

$$
\begin{aligned}
& M_{r}=\max _{t \in J, 0 \leq y \leq r}\{f(t, y)\}>0, \quad M^{*}=\max \left\{M_{k}^{*}, k=1,2, \ldots, n\right\}>0, \\
& M_{k}^{*}=\max _{t \in J, 0 \leq y \leq r}\left\{I_{k}(t, y)\right\}, \quad k=1,2, \ldots, n .
\end{aligned}
$$

Proof If $y \in \partial \Omega_{r}$, then $0 \leq y(t) \leq r$ for $t \in J$.

Since $0 \leq t \leq \alpha(t) \leq 1$ on $J$, it follows from $0 \leq y \leq r$ that

$$
0 \leq y(\alpha(t)) \leq r
$$

Therefore, from $f(t, y) \leq M_{r}$ for $t \in J$ and $y \in \partial \Omega_{r}$, we have

$$
f(t, y(\alpha(t))) \leq M_{r}
$$

So, for $y \in \partial \Omega_{r}$, we have

$$
\begin{aligned}
\left\|T_{\lambda}^{\mu} y\right\|_{P C^{1}} & \leq \rho_{4}\left(\int_{0}^{1} \phi_{m^{*}}\left(\lambda \int_{0}^{1} H(s, \tau) \omega(\tau) f(\tau, y(\alpha(\tau))) d \tau\right) d s+\mu \sum_{k=1}^{n} I_{k}\left(t_{k}, y\left(t_{k}\right)\right)\right) \\
& \leq \rho_{4}\left(\phi_{m^{*}}\left(\lambda \int_{0}^{1} \gamma e(\tau) \omega(\tau) f(\tau, y(\alpha(\tau))) d \tau\right)+\mu \sum_{k=1}^{n} I_{k}\left(t_{k}, y\left(t_{k}\right)\right)\right) \\
& \leq \rho_{4}\left(\phi_{m^{*}}\left(\lambda \gamma \int_{0}^{1} e(\tau) \omega(\tau) M_{r} d \tau\right)+\mu \sum_{k=1}^{n} M^{*}\right) \\
& =\rho_{4}\left(\phi_{m^{*}}\left(\lambda \gamma M_{r} \int_{0}^{1} e(\tau) \omega(\tau) d \tau\right)+\mu n M^{*}\right) \\
& \leq \rho_{4}\left(\phi_{m^{*}}\left(\lambda \gamma M_{r}\|e\|_{q}\|\omega\|_{p}\right)+\mu n M^{*}\right) .
\end{aligned}
$$

This gives the proof.

Corollary 3.3 When $p=1$, assume that $\left(\mathrm{H}_{1}\right)-\left(\mathrm{H}_{4}\right)$ hold and $\alpha(t) \geq t$ on J. If $y \in \partial \Omega_{r}, r>0$, then

$$
\left\|T_{\lambda}^{\mu} y\right\|_{P C^{1}} \leq \rho_{4}\left(\phi_{m^{*}}\left(\lambda \gamma M_{r}\|e\|_{\infty}\|\omega\|_{1}\right)+\mu n M^{*}\right)
$$

Proof By Lemma 3.8, let $\|e\|_{\infty}\|\omega\|_{1}$ replace $\|e\|_{q}\|\omega\|_{p}$ and repeat the argument above.

Corollary 3.4 When $p=\infty$, assume that $\left(\mathrm{H}_{1}\right)-\left(\mathrm{H}_{4}\right)$ hold and $\alpha(t) \geq t$ on J. If $y \in \partial \Omega_{r}$, $r>0$, then

$$
\left\|T_{\lambda}^{\mu} y\right\|_{P C^{1}} \leq \rho_{4}\left(\phi_{m^{*}}\left(\lambda \gamma M_{r}\|e\|_{1}\|\omega\|_{\infty}\right)+\mu n M^{*}\right)
$$

Proof By Lemma 3.8, let $\|e\|_{1}\left\|\omega_{\infty}\right\|$ replace $\|e\|_{q}\left\|\omega_{p}\right\|$ and repeat the argument above.

Lemma 3.9 Assume that $\left(\mathrm{H}_{1}\right)-\left(\mathrm{H}_{4}\right)$ hold and $\alpha(t) \geq t$ on J. If $y \in \partial \Omega_{r}, r>0$, then

$$
\left\|T_{\lambda}^{\mu} y\right\|_{P C^{1}} \geq \rho_{1}\left(\sigma_{r} \lambda \rho \eta\right)^{m^{*}-1} \beta+\mu \rho_{1} n \sigma_{r}^{*}
$$


where

$$
\begin{aligned}
& \sigma_{r}=\min _{t \in J, 0 \leq y \leq r}\{f(t, y)\}>0, \quad \sigma^{*}=\min \left\{m_{k}, k=1,2, \ldots, n\right\}>0, \\
& \sigma_{k}=\min _{t \in J, 0 \leq y \leq r}\left\{I_{k}(t, y)\right\}, \quad k=1,2, \ldots, n .
\end{aligned}
$$

Proof If $y \in \partial \Omega_{r}$, then $0 \leq y(t) \leq r$ for $t \in J$.

Since $0 \leq t \leq \alpha(t) \leq 1$ on $J$, it follows from $0 \leq y(t) \leq r$ for $t \in J$ that

$$
0 \leq y(\alpha(t)) \leq r
$$

Therefore, from $f(t, y) \geq \sigma_{r}$ for $t \in J$ and $0 \leq y \leq r$, we have

$$
f(t, y(\alpha(t))) \geq \sigma_{r}
$$

So, for $y \in \partial \Omega_{r}$, we have

$$
\begin{aligned}
\left\|T_{\lambda}^{\mu} y\right\|_{P C^{1}} & \geq \rho_{1} \phi_{m^{*}}\left(\lambda \int_{0}^{1} \rho e(\tau) \omega(\tau) f(\tau, y(\alpha(\tau))) d \tau\right)+\mu \rho_{1} \sum_{k=1}^{n} I_{k}\left(t_{k}, y\left(t_{k}\right)\right) \\
& \geq \rho_{1}(\lambda \rho \eta)^{m^{*}-1} \phi_{m^{*}}\left(\int_{0}^{1} e(\tau) f(\tau, y(\alpha(\tau))) d \tau\right)+\mu \rho_{1} \sum_{k=1}^{n} I_{k}\left(t_{k}, y\left(t_{k}\right)\right) \\
& \geq \rho_{1}(\lambda \rho \eta)^{m^{*}-1} \phi_{m^{*}}\left(\int_{0}^{1} e(\tau) \sigma_{r} d \tau\right)+\mu \rho_{1} \sum_{k=1}^{n} \sigma_{r}^{*} \\
& \geq \rho_{1}\left(\sigma_{r} \lambda \rho \eta\right)^{m^{*}-1} \phi_{m^{*}}\left(\int_{\zeta}^{1} e(\tau) d \tau\right)+\mu \rho_{1} n \sigma_{r}^{*} \\
& =\rho_{1}\left(\sigma_{r} \lambda \rho \eta\right)^{m^{*}-1} \beta+\mu \rho_{1} n \sigma_{r}^{*} .
\end{aligned}
$$

This finishes the proof.

\section{Main results for the case $\alpha(t) \geq t$ on $J$}

In this section, we apply Lemma 3.2 to establish the existence, multiplicity and nonexistence of positive solutions for problem (1.1). We consider the following three cases for $\omega \in L^{p}[0,1]: p>1, p=1$ and $p=\infty$. Case $p>1$ is treated in the following theorem.

Theorem 4.1 Assume that $\left(\mathrm{H}_{1}\right)-\left(\mathrm{H}_{4}\right)$ hold and $\alpha(t) \geq t$ on J. Then:

(a) If $f^{0}=0$ and $I^{0}(k)=0$ or $f^{\infty}=0$ and $I^{\infty}(k)=0$, then there exist $\lambda_{0}>0$ and $\mu_{0}>0$ such that problem (1.1) has a positive solution for $\lambda>\lambda_{0}$ and $\mu>\mu_{0}$.

(b) If $f_{0}=\infty$ and $I_{0}(k)=\infty$ or $f_{\infty}=\infty$ and $I_{\infty}(k)=\infty$, then there exist $\lambda_{0}>0$ and $\mu_{0}>0$ such that problem (1.1) has a positive solution for $0<\lambda<\lambda_{0}$ and $0<\mu<\mu_{0}$.

(c) If $f^{0}=f^{\infty}=0$ and $I^{0}(k)=I^{\infty}(k)=0$, then there exist $\lambda_{0}>0$ and $\mu_{0}>0$ such that problem (1.1) has at least two positive solutions for $\lambda>\lambda_{0}$ and $\mu>\mu_{0}$.

(d) If $f_{0}=f_{\infty}=\infty$ and $I_{\infty}(k)=I_{\infty}(k)=\infty$, then there exist $\lambda_{0}>0$ and $\mu_{0}>0$ such that problem (1.1) has at least two positive solutions for $0<\lambda<\lambda_{0}$ and $0<\mu<\mu_{0}$.

(e) If $f^{0}<\infty, I^{0}(k)<\infty, f^{\infty}<\infty$ and $I^{\infty}<\infty$, then there exist $\lambda_{0}>0$ and $\mu_{0}>0$ such that problem (1.1) has no positive solution for $0<\lambda<\lambda_{0}$ and $0<\mu<\mu_{0}$. 
(f) If $f_{0}>0, I_{0}(k)>0, I_{\infty}>0$ and $f_{\infty}>0$, then there exist $\lambda_{0}>0$ and $\mu_{0}>0$ such that problem (1.1) has no positive solution for $\lambda>\lambda_{0}$ and $\mu_{0}>0$.

Proof Part (a). Choose a number $r_{1}>0$. By Lemma 3.9, we have $\left\|T_{\lambda}^{\mu} y\right\|_{P C^{1}}>\|y\|_{P C^{1}}$ for $y \in \partial \Omega_{r_{1}}, \lambda>\lambda_{0}$ and $\mu>\mu_{0}$, where

$$
\lambda_{0}=\left(\frac{1}{2 \rho_{1} \beta} r_{1}\right)^{m-1}\left(\sigma_{r} \rho \eta\right)^{-1}>0, \quad \mu_{0}=\frac{1}{2 \rho_{1} n \sigma_{r_{1}}^{*}} r>0 .
$$

If $f^{0}=0$ and $I^{0}(k)=0$, then from Lemma 3.1 we have $f_{0}^{*}=0$ and $I_{0}^{*}(k)=0$, and so we can choose $r_{2} \in\left(0, r_{1}\right)$ so that $f^{*}\left(r_{2}\right) \leq \varepsilon_{1} r_{2}$ and $I_{k}^{*}\left(r_{2}\right) \leq \varepsilon_{2} r_{2}$, where $\varepsilon_{1}>0$ and $\varepsilon_{2}>0$ respectively satisfy

$$
2 \rho_{4} \phi_{m^{*}}\left(\lambda \gamma \varepsilon_{1}\|e\|_{q}\|\omega\|_{p}\right)<1, \quad 2 \rho_{4} \mu n \varepsilon_{2}<1
$$

Then Lemma 3.6 shows that

$$
\left\|T_{\lambda}^{\mu} y\right\|_{P C^{1}} \leq \rho_{4}\left(\phi_{m^{*}}\left(\lambda \gamma \varepsilon_{1}\|e\|_{q}\|\omega\|_{p}\right)+\mu n \varepsilon_{2}\right)\|y\|_{P C^{1}}<\|y\|_{P C^{1}} \quad \text { for } y \in \partial \Omega_{r_{2}} .
$$

If $f^{\infty}=0$ and $I^{\infty}(k)=0$, then from Lemma 3.1, $f_{\infty}^{*}=0$ and $I_{\infty}^{*}(k)=0$. Hence, there exists $r_{3} \in\left(2 r_{1}, \infty\right)$ such that $f^{*}\left(r_{3}\right) \leq \varepsilon_{1} r_{3}$ and $I_{k}^{*}\left(r_{3}\right) \leq \varepsilon_{2} r_{4}$, where $\varepsilon_{1}>0$ and $\varepsilon_{2}>0$ satisfies (4.1). Thus

$$
\left\|T_{\lambda}^{\mu} y\right\|_{P C^{1}} \leq \rho_{4}\left(\phi_{m^{*}}\left(\lambda \gamma \varepsilon_{1}\|e\|_{q}\|\omega\|_{p}\right)+\mu n \varepsilon_{2}\right)\|y\|_{P C^{1}}<\|y\|_{P C^{1}} \quad \text { for } y \in \partial \Omega_{r_{3}} .
$$

Therefore, it follows from Lemma 3.2 that $T_{\lambda}^{\mu}$ has a fixed point in $\bar{\Omega}_{r_{1}} \backslash \Omega_{r_{2}}$ or $\bar{\Omega}_{r_{3}} \backslash \Omega_{r_{1}}$, according to whether $f^{0}=0$ and $I^{0}(k)=0$ or $f^{\infty}=0$ and $I^{\infty}(k)=0$, respectively. Consequently, problem (1.1) has a positive solution for $\lambda>\lambda_{0}$ and $\mu>\mu_{0}$.

Part (b). Choose a number $r_{1}>0$. By Lemma 3.8, there exists $\lambda_{0}>0$ such that

$$
\left\|T_{\lambda}^{\mu} y\right\|_{P C^{1}}<\|y\|_{P C^{1}} \quad \text { for } y \in \partial \Omega_{r_{1}}, 0<\lambda<\lambda_{0} \text { and } 0<\mu<\mu_{0},
$$

where

$$
\lambda_{0}=\left(\frac{1}{2 \rho_{4}}\right)^{m-1}\left(\gamma M_{r}\|e\|_{q}\|\omega\|_{p}\right)^{-1}, \quad \mu_{0}=\frac{1}{2 \rho_{4} n M^{*}} .
$$

If $f_{0}=\infty$ and $I_{0}(k)=\infty$, there exists $r_{2} \in\left(0, r_{1}\right)$ such that $f(t, y) \geq l_{1} \phi_{m}(y)$ and $I_{k}(t, y) \geq l_{2} y$ for $t \in J$ and $0 \leq y \leq r_{2}$, where $l_{1}>0$ and $l_{2}>0$ are chosen so that

$$
2 \delta \frac{\rho_{1}^{2}}{\rho_{4}}\left(l_{1} \lambda \rho \eta\right)^{m^{*}-1} \beta>1, \quad 2 \delta \frac{\rho_{1}^{2}}{\rho_{4}} \mu n l_{2}>1 .
$$

Obviously,

$$
f(t, y) \geq l_{1} \phi_{m}(y), \quad I_{k}(t, y) \geq l_{2} y \quad(k=1,2, \ldots, n) \text { for } y \in \partial \Omega_{r_{2}} \text { and } t \in J
$$


Then, from Lemma 3.7, we can obtain

$$
\left\|T_{\lambda}^{\mu} y\right\|_{P C^{1}} \geq \delta \frac{\rho_{1}^{2}}{\rho_{4}}\left(\left(l_{1} \lambda \rho \eta\right)^{m^{*}-1} \beta+\mu n l_{2}\right)\|y\|_{P C^{1}}>\|y\|_{P C^{1}} \quad \text { for } y \in \partial \Omega_{r_{3}} .
$$

If $f_{\infty}=\infty$ and $I_{\infty}(k)=\infty$, then there exists $\hat{N}>0$ such that $f(t, y) \geq l_{1} \phi_{m}(y), I_{k}(t, y) \geq l_{2} y$ $(k=1,2, \ldots, n)$ for $t \in J$ and $y \geq \hat{N}$, and $l_{1}>0$ and $l_{2}>0$ satisfy (4.2).

Let $r_{3}=\max \left\{2 r_{1}, \hat{N} \rho_{4} / \delta \rho_{1}\right\}$. If $y \in \partial \Omega_{r_{3}}$, then

$$
\min _{t \in[\zeta, 1]} y(t) \geq \frac{\delta \rho_{1}}{\rho_{4}}\|y\|_{P C^{1}} \geq \hat{N}
$$

So,

$$
f(t, y) \geq l_{1} \phi_{m}(y), \quad I_{k}(t, y) \geq l_{2} y \quad(k=1,2, \ldots, n) \text { for } t \in J \text { and } y \in \partial \Omega_{r_{3}} .
$$

From Lemma 3.7, we can get

$$
\left\|T_{\lambda}^{\mu} y\right\|_{P C^{1}} \geq \delta \frac{\rho_{1}^{2}}{\rho_{4}}\left(\left(l_{1} \lambda \rho \eta\right)^{m^{*}-1} \beta+\mu n l_{2}\right)\|y\|_{P C^{1}}>\|y\|_{P C^{1}} \quad \text { for } y \in \partial \Omega_{r_{3}} .
$$

Therefore, it follows from Lemma 3.2 that $T_{\lambda}^{\mu}$ has a fixed point in $\bar{\Omega}_{r_{1}} \backslash \Omega_{r_{2}}$ or $\bar{\Omega}_{r_{3}} \backslash \Omega_{r_{1}}$, according to whether $f_{0}=\infty$ and $I_{0}(k)=\infty$ or $f_{\infty}=\infty$ and $I_{\infty}(k)=\infty$, respectively. Consequently, problem (1.1) has a positive solution for $0<\lambda<\lambda_{0}$ and $0<\mu<\mu_{0}$.

Part (c). Choose two numbers $0<r_{3}<r_{4}$. By Lemma 3.9, there exist $\lambda_{0}>0$ and $\mu_{0}>0$ such that

$$
\left\|T_{\lambda}^{\mu} y\right\|_{P C^{1}}>\|y\|_{P C^{1}} \quad \text { for } y \in \partial \Omega_{r_{i}}, i=3,4
$$

Since $f^{0}=f^{\infty}=0$ and $I^{0}(k)=I^{\infty}(k)=0$, from the proof of part (a), it follows that we can choose $r_{1} \in\left(0, r_{3} / 2\right)$ and $r_{2} \in\left(2 r_{4}, \infty\right)$ such that

$$
\left\|T_{\lambda}^{\mu} y\right\|_{P C^{1}}<\|y\|_{P C^{1}} \quad \text { for } y \in \partial \Omega_{r_{i}}, i=1,2
$$

It follows from Lemma 3.2 that $T_{\lambda}^{\mu}$ has two fixed points $y_{1}$ and $y_{2}$ such that $y_{1} \in \bar{\Omega}_{r_{3}} \backslash \Omega_{r_{1}}$ and $y_{2} \in \bar{\Omega}_{r_{2}} \backslash \Omega_{r_{4}}$. These are the desired distinct positive solutions of problem (1.1) for $\lambda>\lambda_{0}$ and $\mu>\mu_{0}$ satisfying

$$
r_{1} \leq\left\|y_{1}\right\|_{P C^{1}} \leq r_{3}<r_{4} \leq\left\|y_{2}\right\|_{P C^{1}} \leq r_{2}
$$

Part (d). Choose two numbers $0<r_{3}<r_{4}$. By Lemma 3.8, there exist $\lambda_{0}>0$ and $\mu_{0}>0$ such that

$$
\left\|T_{\lambda}^{\mu} y\right\|_{P C^{1}}<\|y\|_{P C^{1}} \quad \text { for } 0<\lambda<\lambda_{0}, 0<\mu<\mu_{0} \text { and } y \in \partial \Omega_{r_{i}}, i=3,4
$$

Since $f_{0}=\infty$ and $f_{\infty}=\infty$ and $I_{\infty}(k)=I_{\infty}(k)=\infty$, from the proof of part (b), we know that we can choose $r_{1} \in\left(0, r_{3} / 2\right)$ and $r_{2} \in\left(2 r_{4}, \infty\right)$ such that

$$
\left\|T_{\lambda}^{\mu} y\right\|_{P C^{1}}>\|y\|_{P C^{1}} \quad \text { for } y \in \partial \Omega_{r_{i}}, i=1,2
$$


It follows from Lemma 3.2 that $T_{\lambda}^{\mu}$ has two fixed points $y_{1}$ and $y_{2}$ such that $y_{1} \in \bar{\Omega}_{r_{3}} \backslash \Omega_{r_{1}}$ and $y_{2} \in \bar{\Omega}_{r_{2}} \backslash \Omega_{r_{4}}$. These are the desired distinct positive solutions of problem (1.1) for $0<\lambda<\lambda_{0}$ and $0<\mu<\mu_{0}$ satisfying (4.3).

Part (e). Since $f^{0}<\infty, I^{0}(k)<\infty, f^{\infty}<\infty$ and $I^{\infty}(k)<\infty$, there exist positive numbers $l_{i}>0(i=1,2,3,4), h_{1}>0$ and $h_{2}>0$ such that $h_{1}<h_{2}$ and for $t \in J, 0<y \leq h_{1}$, we have

$$
f(t, y) \leq l_{1} \phi_{m}(y), \quad I_{k}(t, y) \leq l_{2} y
$$

and for $t \in J, y \geq h_{2}$, we have

$$
f(t, y) \leq l_{3} \phi_{m}(y), \quad I_{k}(t, y) \leq l_{4} y .
$$

Let

$$
\begin{aligned}
& l=\max \left\{l_{1}, l_{3}, \max \left\{\frac{f(t, y)}{\phi_{m}(y)}: t \in J, h_{1} \leq y \leq h_{2}\right\}\right\}>0, \\
& l^{*}=\max \left\{l_{2}, l_{4}, \max \left\{\frac{I_{k}(t, y)}{y}: t \in J, h_{1} \leq y \leq h_{2}\right\}\right\}>0 .
\end{aligned}
$$

Thus, we have

$$
f(t, y) \leq l \phi_{m}(y), \quad I_{k}(t, y) \leq l^{*} y \quad \text { for } t \in J \text { and } y \in[0, \infty)
$$

Since $0 \leq t \leq \alpha(t) \leq 1$ on $J$, it follows from $0 \leq y(t) \leq h_{1}, y(t) \geq h_{2}$ and $h_{1} \leq y(t) \leq h_{2}$ on $J$ that $0 \leq y(\alpha(t)) \leq h_{1}, y(\alpha(t)) \geq h_{2}$ and $h_{1} \leq y(\alpha(t)) \leq h_{2}$ on $J$, respectively.

Assume that $y$ is a positive solution of problem (1.1). We will show that this leads to a contradiction for

$$
0<\lambda<\lambda_{0}=\left(\frac{1}{2 \rho_{4}}\right)^{m-1}\left(\gamma l\|e\|_{q}\|\omega\|_{p}\right)^{-1}
$$

and

$$
0<\mu<\mu_{0}=\frac{1}{2 \rho_{4} n l^{*}} .
$$

Since $\left(T_{\lambda}^{\mu} y\right)(t)=y(t)$ for $t \in J$, by Lemma 3.6 we have that

$$
\|y\|_{P C^{1}}=\left\|T_{\lambda}^{\mu} y\right\|_{P C^{1}} \leq \rho_{4}\left(\phi_{m^{*}}\left(\lambda \gamma l\|e\|_{q}\|\omega\|_{p}\right)+\mu n l^{*}\right)\|y\|_{P C^{1}}<\|y\|_{P C^{1}}
$$

which is a contradiction.

Part (f). Since $f_{0}>0, I_{0}(k)>0, I_{\infty}>0$ and $f_{\infty}>0$, there exist positive numbers $l_{i}>0$ $(i=5,6,7,8), h_{3}>0$ and $h_{4}>0$ such that $h_{3}<h_{4}$ and for $t \in J, 0 \leq y \leq h_{3}$, we have

$$
f(t, y) \geq l_{5} \phi_{m}(y), \quad I_{k}(t, y) \geq l_{6} y
$$

and for $t \in J, y \geq h_{4}$, we have

$$
f(t, y) \geq l_{7} \phi_{m}(y), \quad I_{k}(t, y) \geq l_{8} y .
$$


Let

$$
\begin{aligned}
& l^{* *}=\min \left\{l_{5}, l_{7}, \min \left\{\frac{f(t, y)}{\phi_{m}(y)}: t \in J, h_{3} \leq y \leq h_{4}\right\}\right\}>0, \\
& l^{* * *}=\min \left\{l_{6}, l_{8}, \min \left\{\frac{I_{k}(t, y)}{y}: t \in J, h_{3} \leq y \leq h_{4}\right\}\right\}>0 .
\end{aligned}
$$

Then

$$
f(t, y) \geq l^{* *} \phi_{m}(y), \quad I_{k}(t, y) \geq l^{* * *} y \quad \text { for } t \in J \text { and } y \in[0, \infty) .
$$

Assume that $y$ is a positive solution of problem (1.1). We will show that this leads to a contradiction for

$$
\begin{aligned}
& \lambda>\lambda_{0}=\left(\frac{\rho_{4}}{2 \delta \rho_{1}^{2} \beta}\right)^{m-1}\left(l^{* *} \rho \eta\right)^{-1}, \\
& \mu>\mu_{0}=\frac{\rho_{4}}{2 \delta \rho_{1}^{2} \eta l^{* * *}} .
\end{aligned}
$$

Since $\left(T_{\lambda}^{\mu} y\right)(t)=y(t)$ for $t \in J$, by Lemma 3.7 we have that

$$
\|y\|_{P C^{1}}=\left\|T_{\lambda}^{\mu} y\right\|_{P C^{1}} \geq \delta \frac{\rho_{1}^{2}}{\rho_{4}}\left(\left(l^{* *} \lambda \rho \eta\right)^{m^{*}-1} \beta+\mu n l^{* * *}\right)\|y\|_{P C^{1}}>\|y\|_{P C^{1}},
$$

which is a contradiction.

The results of the following theorem deal with the case $p=1$.

Corollary 4.1 Assume that $\left(\mathrm{H}_{1}\right)-\left(\mathrm{H}_{4}\right)$ hold and $\alpha(t) \geq t$ on $J$. Then:

(a) If $f^{0}=0$ and $I^{0}(k)=0$ or $f^{\infty}=0$ and $I^{\infty}(k)=0$, then there exist $\lambda_{0}>0$ and $\mu_{0}>0$ such that problem (1.1) has a positive solution for $\lambda>\lambda_{0}$ and $\mu>\mu_{0}$.

(b) If $f_{0}=\infty$ and $I_{0}(k)=\infty$ or $f_{\infty}=\infty$ and $I_{\infty}(k)=\infty$, then there exist $\lambda_{0}>0$ and $\mu_{0}>0$ such that problem (1.1) has a positive solution for $0<\lambda<\lambda_{0}$ and $0<\mu<\mu_{0}$.

(c) If $f^{0}=f^{\infty}=0$ and $I^{0}(k)=I^{\infty}(k)=0$, then there exist $\lambda_{0}>0$ and $\mu_{0}>0$ such that problem (1.1) has at least two positive solutions for $\lambda>\lambda_{0}$ and $\mu>\mu_{0}$.

(d) If $f_{0}=f_{\infty}=\infty$ and $I_{\infty}(k)=I_{\infty}(k)=\infty$, then there exist $\lambda_{0}>0$ and $\mu_{0}>0$ such that problem (1.1) has at least two positive solutions for $0<\lambda<\lambda_{0}$ and $0<\mu<\mu_{0}$.

(e) If $f^{0}<\infty, I^{0}(k)<\infty, f^{\infty}<\infty$ and $I^{\infty}<\infty$, then there exist $\lambda_{0}>0$ and $\mu_{0}>0$ such that problem (1.1) has no positive solution for $0<\lambda<\lambda_{0}$ and $0<\mu<\mu_{0}$.

(f) If $f_{0}>0, I_{0}(k)>0, I_{\infty}>0$ and $f_{\infty}>0$, then there exist $\lambda_{0}>0$ and $\mu_{0}>0$ such that problem (1.1) has no positive solution for $\lambda>\lambda_{0}$ and $\mu_{0}>0$.

Proof It follows from the proofs of Corollary 3.1 and Corollary 3.3 that Corollary 4.1 holds.

Finally we consider the case of $p=\infty$.

Corollary 4.2 Assume that $\left(\mathrm{H}_{1}\right)-\left(\mathrm{H}_{4}\right)$ hold and $\alpha(t) \geq t$ on $J$. Then: 
(a) If $f^{0}=0$ and $I^{0}(k)=0$ or $f^{\infty}=0$ and $I^{\infty}(k)=0$, then there exist $\lambda_{0}>0$ and $\mu_{0}>0$ such that problem (1.1) has a positive solution for $\lambda>\lambda_{0}$ and $\mu>\mu_{0}$.

(b) If $f_{0}=\infty$ and $I_{0}(k)=\infty$ or $f_{\infty}=\infty$ and $I_{\infty}(k)=\infty$, then there exist $\lambda_{0}>0$ and $\mu_{0}>0$ such that problem (1.1) has a positive solution for $0<\lambda<\lambda_{0}$ and $0<\mu<\mu_{0}$.

(c) If $f^{0}=f^{\infty}=0$ and $I^{0}(k)=I^{\infty}(k)=0$, then there exist $\lambda_{0}>0$ and $\mu_{0}>0$ such that problem (1.1) has at least two positive solutions for $\lambda>\lambda_{0}$ and $\mu>\mu_{0}$.

(d) If $f_{0}=f_{\infty}=\infty$ and $I_{\infty}(k)=I_{\infty}(k)=\infty$, then there exist $\lambda_{0}>0$ and $\mu_{0}>0$ such that problem (1.1) has at least two positive solutions for $0<\lambda<\lambda_{0}$ and $0<\mu<\mu_{0}$.

(e) If $f^{0}<\infty, I^{0}(k)<\infty, f^{\infty}<\infty$ and $I^{\infty}<\infty$, then there exist $\lambda_{0}>0$ and $\mu_{0}>0$ such that problem (1.1) has no positive solution for $0<\lambda<\lambda_{0}$ and $0<\mu<\mu_{0}$.

(f) If $f_{0}>0, I_{0}(k)>0, I_{\infty}>0$ and $f_{\infty}>0$, then there exist $\lambda_{0}>0$ and $\mu_{0}>0$ such that problem (1.1) has no positive solution for $\lambda>\lambda_{0}$ and $\mu_{0}>0$.

Proof It follows from the proofs of Corollary 3.2 and Corollary 3.4 that Corollary 4.2 holds.

\section{Positive solutions of problem (1.1) for the case of $\alpha(t) \leq t$ on $J$}

Now we deal with problem (1.1) for the case of $\alpha(t) \leq t$ on $J$. Similarly as Theorem 2.4 and Lemmas 3.3-3.9, we can prove the following results.

Lemma 5.1 Let $\zeta^{*} \in\left(t_{n}, 1\right), G_{1}$ and $H_{1}$ be given as in Theorem 2.3. If $\left(\mathrm{H}_{4}\right)$ holds, then we have

$$
G_{1}(t, s) \geq \delta G_{1}(s, s) \geq \frac{b^{2} \delta}{d}, \quad H_{1}(t, s) \geq \frac{\delta a}{a-\xi} G_{1}(s, s) \geq \delta \rho_{1}, \quad \forall t \in\left[0, \zeta^{*}\right], s \in J,
$$

where $d$ is defined in Theorem 2.3, $\delta$ and $\rho_{1}$ are defined in (2.8).

Proof In fact, for $t \in\left[0, \zeta^{*}\right]$ and $s \in J$, we have that

$$
\begin{aligned}
& \frac{G_{1}(t, s)}{G_{1}(s, s)}=\frac{b+a(1-t)}{b+a(1-s)} \geq \frac{b+a\left(1-\zeta^{*}\right)}{b+a} \quad \text { for } s \leq t, \\
& \frac{G_{1}(t, s)}{G_{1}(s, s)}=\frac{b+a t}{b+a s} \geq \frac{b}{b+a} \quad \text { for } t \leq s .
\end{aligned}
$$

This and (2.13) show that

$$
\begin{aligned}
H_{1}(t, s) & \geq \delta G_{1}(s, s)\left[1+\frac{1}{a-\xi} \int_{0}^{1} g(\tau) d \tau\right] \\
& \geq \frac{a \delta}{a-\xi} G_{1}(s, s), \quad t \in\left[0, \zeta^{*}\right], s \in J .
\end{aligned}
$$

This together with (2.13) and (2.15) finishes the proof of (5.1).

Let $P C^{1}[0,1]$ be as defined in Section 3 . We define a cone $K^{*}$ in $P C^{1}[0,1]$ by

$$
K^{*}=\left\{y \in P C^{1}[0,1] \mid y(t) \geq 0 \text { on } J \text { and } \min _{t \in\left[0, \zeta^{*}\right]} \geq \delta \frac{\rho_{1}}{\rho_{4}}\|y\|_{P C^{1}}\right\},
$$

where $\delta, \rho_{1}$ and $\rho_{4}$ are defined in (2.18) and (3.8), respectively. It is easy to see that $K^{*}$ is a closed convex cone of $P C^{1}[0,1]$. 
Define $^{*} T_{\lambda}^{\mu}: K^{*} \rightarrow P C^{1}[0,1]$ by

$$
\begin{aligned}
\left({ }^{*} T_{\lambda}^{\mu} y\right)(t)= & \int_{0}^{1} H_{1}(t, s) \phi_{m^{*}}\left(\lambda \int_{0}^{1} H(s, \tau) \omega(\tau) f(\tau, y(\alpha(\tau))) d \tau\right) d s \\
& +\mu \sum_{k=1}^{n} H_{1}\left(t, t_{k}\right) I_{k}\left(t_{k}, y\left(t_{k}\right)\right) .
\end{aligned}
$$

It is clear that $y$ is a positive solution of problem (1.1) if and only of $y$ is a fixed point of ${ }^{*} T_{\lambda}^{\mu}$.

Lemma 5.2 Assume that $\left(\mathrm{H}_{1}\right)-\left(\mathrm{H}_{4}\right)$ hold. Then $y \in K^{*}$ is a positive fixed point of ${ }^{*} T_{\lambda}^{\mu}$ if and only if $y$ is a positive solution of problem (1.1).

Lemma 5.3 Assume that $\left(\mathrm{H}_{1}\right)-\left(\mathrm{H}_{4}\right)$ hold. Then ${ }^{*} T_{\lambda}^{\mu}\left(K^{*}\right) \subset K^{*}$ and ${ }^{*} T_{\lambda}^{\mu}: K^{*} \rightarrow K^{*}$ is completely continuous.

Let $f^{*}$ and $I_{k}^{*}$ be defined as in Section 3. Similar to the proof of that in Lemmas 3.6-3.9, we have the following results. Here, we only consider the case $m>1$ and only give the proof of Lemma 5.4 .

Lemma 5.4 Assume that $\left(\mathrm{H}_{1}\right)-\left(\mathrm{H}_{4}\right)$ hold, $\alpha(t) \leq t$ on $J$ and let $r>0$ be given. If there exist $\varepsilon_{1}>0$ and $\varepsilon_{2}>0$ such that $f^{*}(r) \leq \varepsilon_{1} \phi_{m}(r)$ and $I_{k}^{*}(r) \leq \varepsilon_{2} r(k=1,2, \ldots, n)$, then

$$
\left\|T^{*} T_{\lambda}^{\mu} y\right\| \leq \rho_{4}\left(\phi_{m^{*}}\left(\lambda \gamma \varepsilon_{1}\|e\|_{q}\|\omega\|_{p}\right)+\mu n \varepsilon_{2}\right)\|y\|_{P C^{1}}, \quad y \in \partial \Omega_{r} .
$$

Proof By the definition of $f^{*}(r)$ and $I_{k}^{*}$, if $f^{*}(r) \leq \varepsilon_{1} \phi_{m}(r)$ and $I_{k}^{*}(r) \leq \varepsilon_{2} r(k=1,2, \ldots, n)$, then

$$
f(t, y) \leq \varepsilon_{1} \phi_{m}(r), \quad I_{k}(t, y) \leq \varepsilon_{2} r \quad \text { for } t \in J \text { and } 0 \leq y \leq r .
$$

Since $0 \leq \alpha(t) \leq t \leq 1$ on $J$, it follows from $0 \leq y(t) \leq r$ on $J$ that $0 \leq y(\alpha(t)) \leq r$.

Therefore, we have $f(t, y(\alpha(t))) \leq \varepsilon_{1} \phi_{m}(r)$ for $t \in J$, and it follows from (3.7) and (5.2) that

$$
\begin{aligned}
\left\|^{*} T_{\lambda}^{\mu} y\right\|_{P C^{1}} & \leq \rho_{4}\left(\int_{0}^{1} \phi_{m^{*}}\left(\lambda \int_{0}^{1} H(s, \tau) \omega(\tau) f(\tau, y(\alpha(\tau))) d \tau\right) d s+\mu \sum_{k=1}^{n} I_{k}\left(t_{k}, y\left(t_{k}\right)\right)\right) \\
& \leq \rho_{4}\left(\phi_{m^{*}}\left(\lambda \int_{0}^{1} \gamma e(\tau) \omega(\tau) f(\tau, y(\alpha(\tau))) d \tau\right)+\mu \sum_{k=1}^{n} I_{k}\left(t_{k}, y\left(t_{k}\right)\right)\right) \\
& \leq \rho_{4}\left(\phi_{m^{*}}\left(\lambda \gamma \int_{0}^{1} e(\tau) \omega(\tau) \varepsilon_{1} \phi_{m}(r) d \tau\right)+\mu \sum_{k=1}^{n} \varepsilon_{2} r\right) \\
& =\rho_{4}\left(\phi_{m^{*}}\left(\lambda \gamma \varepsilon_{1} \phi_{m}(r) \int_{0}^{1} e(\tau) \omega(\tau) d \tau\right)+\mu \sum_{k=1}^{n} \varepsilon_{2} r\right) \\
& \leq \rho_{4}\left(\phi_{m^{*}}\left(\lambda \gamma \varepsilon_{1} \phi_{m}(r)\|e\|_{q}\|\omega\|_{p}\right)+\mu n \varepsilon_{2} r\right) \\
& =\rho_{4}\left(\phi_{m^{*}}\left(\lambda \gamma \varepsilon_{1}\|e\|_{q}\|\omega\|_{p}\right)+\mu n \varepsilon_{2} r\right)\|y\|_{P C^{1}}, \quad \forall y \in \partial \Omega_{r} .
\end{aligned}
$$

This completes the proof. 
Lemma 5.5 Assume that $\left(\mathrm{H}_{1}\right)-\left(\mathrm{H}_{4}\right)$ hold, $\alpha(t) \leq t$ on $J$ and let $l_{1}>0$ and $l_{2}$ be given. If $f(t, y) \geq l_{1} \phi_{m}(y)$ and $I_{k}(t, y) \geq l_{2} y$ for $t \in J$ and $y \in K^{*}$, then

$$
\left\|{ }^{*} T_{\lambda}^{\mu} y\right\|_{P C^{1}} \geq \delta \frac{\rho_{1}^{2}}{\rho_{4}}\left(\left(l_{1} \lambda \rho \eta\right)^{m^{*}-1} \beta+\mu n l_{2}\right)\|y\|_{P C^{1}} .
$$

Let $\hat{\Omega}_{r}=\left\{y \in K^{*}:\|y\|_{P C^{1}}<r\right\}$.

Lemma 5.6 Assume that $\left(\mathrm{H}_{1}\right)-\left(\mathrm{H}_{4}\right)$ hold and $\alpha(t) \leq t$ on J. If $y \in \partial \hat{\Omega}_{r}, r>0$, then

$$
\left\|^{*} T_{\lambda}^{\mu} y\right\|_{P C^{1}} \leq \rho_{4}\left(\phi_{m^{*}}\left(\lambda \gamma M_{r}\|e\|_{q}\|\omega\|_{p}\right)+\mu n M^{*}\right)
$$

where $M_{r}$ and $M^{*}$ are defined in Lemma 3.8.

Lemma 5.7 Assume that $\left(\mathrm{H}_{1}\right)-\left(\mathrm{H}_{4}\right)$ hold and $\alpha(t) \leq t$ on J. If $y \in \partial \hat{\Omega}_{r}, r>0$, then

$$
\left\|^{*} T_{\lambda} u\right\|_{P C^{1}} \geq \rho_{1}\left(\sigma_{r} \lambda \rho \eta\right)^{m^{*}-1} \beta+\mu \rho_{1} n \sigma_{r}^{*}
$$

where $\sigma_{r}$ and $\sigma^{*}$ are defined in Lemma 3.9.

Let $f^{0}, f^{\infty}, f_{0}, f_{\infty}, I^{0}(k), I^{\infty}(k), I_{0}(k)$ and $I_{\infty}(k)$ be defined as in Section 3. Similar to the proof of Theorem 4.1, we have the following results.

Theorem 5.1 Assume that $\left(\mathrm{H}_{1}\right)-\left(\mathrm{H}_{4}\right)$ hold and $\alpha(t) \leq t$ on J. Then:

(a) If $f^{0}=0$ and $I^{0}(k)=0$ or $f^{\infty}=0$ and $I^{\infty}(k)=0$, then there exist $\lambda_{0}>0$ and $\mu_{0}>0$ such that problem (1.1) has a positive solution for $\lambda>\lambda_{0}$ and $\mu>\mu_{0}$.

(b) If $f_{0}=\infty$ and $I_{0}(k)=\infty$ or $f_{\infty}=\infty$ and $I_{\infty}(k)=\infty$, then there exist $\lambda_{0}>0$ and $\mu_{0}>0$ such that problem (1.1) has a positive solution for $0<\lambda<\lambda_{0}$ and $0<\mu<\mu_{0}$.

(c) If $f^{0}=f^{\infty}=0$ and $I^{0}(k)=I^{\infty}(k)=0$, then there exist $\lambda_{0}>0$ and $\mu_{0}>0$ such that problem (1.1) has at least two positive solutions for $\lambda>\lambda_{0}$ and $\mu>\mu_{0}$.

(d) If $f_{0}=f_{\infty}=\infty$ and $I_{\infty}(k)=I_{\infty}(k)=\infty$, then there exist $\lambda_{0}>0$ and $\mu_{0}>0$ such that problem (1.1) has at least two positive solutions for $0<\lambda<\lambda_{0}$ and $0<\mu<\mu_{0}$.

(e) If $f^{0}<\infty, I^{0}(k)<\infty, f^{\infty}<\infty$ and $I^{\infty}<\infty$, then there exist $\lambda_{0}>0$ and $\mu_{0}>0$ such that problem (1.1) has no positive solution for $0<\lambda<\lambda_{0}$ and $0<\mu<\mu_{0}$.

(f) If $f_{0}>0, I_{0}(k)>0, I_{\infty}>0$ and $f_{\infty}>0$, then there exist $\lambda_{0}>0$ and $\mu_{0}>0$ such that problem (1.1) has no positive solution for $\lambda>\lambda_{0}$ and $\mu_{0}>0$.

\section{Remarks and comments}

In this section, we offer some remarks and comments of the associated problem (1.1).

Remark 6.1 The idea of deviating arguments for problem (1.1) is from Jankowski [34], but the method and conclusion are quite different, and Jankowski only considered the case $\lambda=1, \mu=1$ and $\omega \in C[0,1]$, not $\omega(t)$ is $L^{p}$-integrable.

Remark 6.2 Generally, it is difficult to study the existence of positive solutions for nonlinear fourth order boundary value problems with impulsive effects and deviating arguments (see, e.g., [15-25] and their references). 
For example, we consider the following problems:

$$
\left\{\begin{array}{l}
\left(\phi_{m}\left(y^{\prime \prime}(t)\right)\right)^{\prime \prime}=\lambda \omega(t) f(t, y(\alpha(t))), \quad t \in J, t \neq t_{k}, k=1,2, \ldots, n, \\
\left.\Delta y^{\prime}\right|_{t=t_{k}}=-\mu I_{k}\left(t_{k}, y\left(t_{k}\right)\right), \quad k=1,2, \ldots, n, \\
y(0)=0, \quad y(1)=\int_{0}^{1} g(t) y(t) d t \\
\phi_{p}\left(y^{\prime \prime}(0)\right)=0, \quad \phi_{p}\left(y^{\prime \prime}(1)\right)=\int_{0}^{1} h(t) \phi_{p}\left(y^{\prime \prime}(t)\right) d t
\end{array}\right.
$$

Here $\lambda>0$ and $\mu>0$ are two parameters, $a, b>0, J=[0,1], \phi_{m}(s)$ is an $m$-Laplace operator, i.e., $\phi_{m}(s)=|s|^{m-2} s, m>1,\left(\phi_{m}\right)^{-1}=\phi_{m^{*}}, \frac{1}{m}+\frac{1}{m^{*}}=1, t_{k}(k=1,2, \ldots, n)$ (where $n$ is a fixed positive integer) are fixed points with $0=t_{0}<t_{1}<t_{2}<\cdots<t_{k}<\cdots<t_{n}<t_{n+1}=1,\left.\Delta y^{\prime}\right|_{t=t_{k}}=$ $y^{\prime}\left(t_{k}^{+}\right)-x^{\prime}\left(t_{k}^{-}\right)$, where $y^{\prime}\left(t_{k}^{+}\right)$and $y^{\prime}\left(t_{k}^{-}\right)$represent the right-hand limit and the left-hand limit of $y^{\prime}(t)$ at $t=t_{k}$, respectively.

By means of transformation (2.2), we can convert problem (4.1) into

$$
\left\{\begin{array}{l}
x^{\prime \prime}(t)+\lambda \omega(t) f(t, y(\alpha(t)))=0, \quad t \in J \\
x(0)=0, \quad x(1)=\int_{0}^{1} h(t) x(t) d t
\end{array}\right.
$$

and

$$
\left\{\begin{array}{l}
y^{\prime \prime}(t)=-\phi_{m^{*}}(x(t)), \quad t \in J, t \neq t_{k}, \\
\left.\Delta y^{\prime}\right|_{t=t_{k}}=-\mu I_{k}\left(t_{k}, y\left(t_{k}\right)\right), \quad k=1,2, \ldots, n, \\
y(0)=0, \quad y(1)=\int_{0}^{1} g(t) y(t) d t .
\end{array}\right.
$$

Using a similar proof to that of Theorem 2.1 and Theorem 2.2, we can obtain the following results. In addition, if we replace $\xi, v$ by $\xi^{*}, v^{*}$ in $\left(\mathrm{H}_{2}\right)$, respectively, then we obtain $\left(\mathrm{H}_{2}^{*}\right)$, where

$$
\xi^{*}=\int_{0}^{1} s g(s) d s, \quad v^{*}=\int_{0}^{1} \operatorname{sh}(s) d s .
$$

Lemma 6.1 If $\left(\mathrm{H}_{1}\right),\left(\mathrm{H}_{2}^{*}\right)$ and $\left(\mathrm{H}_{4}\right)$ hold, then problem (6.2) has a unique solution $x$ expressed by

$$
x(t)=\lambda \int_{0}^{1} H^{*}(t, s) f(s, y(s)) d s
$$

where

$$
H^{*}(t, s)=G(t, s)+\frac{t}{1-\int_{0}^{1} \operatorname{sh}(s) d s} \int_{0}^{1} G(\tau, s) h(\tau) d \tau
$$

$G(t, s)$ is defined in (2.6).

Lemma 6.2 If $\left(\mathrm{H}_{1}\right),\left(\mathrm{H}_{3}\right)$ and $\left(\mathrm{H}_{4}\right)$ hold, then problem (6.3) has a unique solution y given by

$$
y(t)=\int_{0}^{1} H_{1}^{*}(t, s) \phi_{m^{*}}(x(s)) d s+\sum_{k=1}^{n} H_{1}^{*}\left(t, t_{k}\right) I_{k}\left(t_{k}, y\left(t_{k}\right)\right)
$$


where

$$
H_{1}^{*}(t, s)=G(t, s)+\frac{t}{1-\int_{0}^{1} s g(s) d s} \int_{0}^{1} G(s, \tau) g(\tau) d \tau .
$$

It is not difficult to prove that $H^{*}(t, s)$ and $H_{1}^{*}(t, s)$ have similar properties to those of $H(t, s)$ and $H_{1}(t, s)$. However, there does not exist a positive number $\delta \in(0,1)$ such that

$$
G(t, s) \geq \delta G(s, s) \quad \text { for } t \in[\zeta, 1], s \in J
$$

or there does not exist a positive number $\delta^{*} \in(0,1)$ such that

$$
G(t, s) \geq \delta^{*} G(s, s) \text { for } t \in\left[0, \zeta^{*}\right], s \in J,
$$

where $\zeta \in\left(0, t_{1}\right)$ and $\zeta^{*} \in\left(t_{n}, 1\right)$. This implies that we cannot study the existence of positive solutions for problem (6.1) when the deviating arguments are delayed and advanced.

Remark 6.3 There are many functions $\alpha(t)$ satisfying $\alpha(t) \geq t$ or $\alpha(t) \leq t$ on $J$. For example,

$$
\begin{aligned}
& \text { if } \alpha(t)=t^{\frac{1}{n}}, \quad \text { then } \alpha(t) \geq t \text { on } J ; \\
& \text { if } \alpha(t)=t^{n}, \quad \text { then } \alpha(t) \leq t \text { on } J,
\end{aligned}
$$

where $n$ is a positive integral number.

\section{Competing interests}

The authors declare that they have no competing interests.

\section{Authors' contributions}

MF checked the proofs and verified the calculation. JQ completed the main study and carried out the results of this article. All the authors read and approved the final manuscript.

\section{Acknowledgements}

We wish to express our thanks to Prof. Xuemei Zhang, Department of Mathematics and Physics, North China Electric Power University, Beijing, P.R. China, for her kind help, careful reading, and making useful comments on the earlier version of this paper. The authors also thank the project NSFC (11171032) and the improving project of graduate education of Beijing Information Science and Technology University (YJT201416) for their support.

Received: 20 June 2014 Accepted: 3 February 2015 Published online: 20 February 2015

\section{References}

1. Kuang, Y: Delay Differential Equations: With Applications in Population Dynamics. Academic Press, Boston (1993)

2. Yan, JR: Existence and global attractivity of positive periodic solution for an impulsive Lasota-Wazewska model. J. Math. Anal. Appl. 279, 111-120 (2003)

3. Gyöi, I, Ladas, G: Oscillation Theorem of Delay Differential Equations with Applications. Clarendon, Oxford (1991)

4. Lalli, BS, Zhang, BG: On a periodic delay population model. Q. Appl. Math. 52, 35-42 (1994)

5. Gopalsamy, K, Kulenović, MRS, Ladas, G: Environmental periodicity and time delays in a food-limited population model. J. Math. Anal. Appl. 147, 545-555 (1990)

6. Zhang, XM, Feng, MQ: Transformation technique, fixed point theorem and positive solutions for second-order impulsive differential equations with deviating arguments. Adv. Differ. Equ. 2014, 312 (2014)

7. Nieto, JJ, Rodríguez-López, R: Periodic boundary value problem for non-Lipschitzian impulsive functional differential equations. J. Math. Anal. Appl. 318, 593-610 (2006)

8. Yan, JR, Shen, JH: Impulsive stabilization of functional differential equations by Lyapunov-Razumikhin functions. Nonlinear Anal. 37, 245-255 (1999)

9. Li, JL, Shen, JH: New comparison results for impulsive functional differential equations. Appl. Math. Lett. 23, 487-493 (2010) 
10. Yang, XX, Shen, JH: Nonlinear boundary value problems for first-order impulsive functional differential equations. Appl. Math. Comput. 189, 1943-1952 (2007)

11. Liu, YS: Periodic boundary value problems for first order functional differential equations with impulse. J. Comput. Appl. Math. 223, 27-39 (2009)

12. Liu, YJ: Further results on periodic boundary value problems for nonlinear first-order impulsive functional differential equations. J. Math. Anal. Appl. 327, 435-452 (2007)

13. He, ZM, Yu, JS: Periodic boundary value problem for first-order impulsive functional differential equations. J. Math. Anal. Appl. 272, 67-78 (2002)

14. Ding, W, Han, MA, Mi, JR: Periodic boundary value problem for the second-order impulsive functional differential equations. Comput. Math. Appl. 50, 491-507 (2005)

15. Sun, JP, Wang, XQ: Monotone positive solutions for an elastic beam equation with nonlinear boundary conditions. Math. Probl. Eng. 2011, Article ID 609189 (2011)

16. Yao, QL: Positive solutions of nonlinear beam equations with time and space singularities. J. Math. Anal. Appl. 374 681-692 (2011)

17. O'Regan, D: Solvability of some fourth (and higher) order singular boundary value problems. J. Math. Anal. Appl. 161, 78-116 (1991)

18. Yang, B: Positive solutions for the beam equation under certain boundary conditions. Electron. J. Differ. Equ. 2005, 78 (2005)

19. Zhang, XG: Existence and iteration of monotone positive solutions for an elastic beam equation with a corner. Nonlinear Anal., Real World Appl. 10, 2097-2103 (2009)

20. Gupta, GP: Existence and uniqueness theorems for the bending of an elastic beam equation. Appl. Anal. 26, 289-304 (1988)

21. Agarwal, RP: On fourth-order boundary value problems arising in beam analysis. Differ. Integral Equ. 2, 91-110 (1989)

22. Bonanno, G, Bella, BD: A boundary value problem for fourth-order elastic beam equations. J. Math. Anal. Appl. 343, $1166-1176(2008)$

23. Han, GD, Xu, ZB: Multiple solutions of some nonlinear fourth-order beam equations. Nonlinear Anal. TMA 68 3646-3656 (2008)

24. Zhang, XG, Liu, LS: Positive solutions of fourth-order four-point boundary value problems with $p$-Laplacian operator. J. Math. Anal. Appl. 336, 1414-1423 (2007)

25. Feng, MQ: Multiple positive solutions of four-order impulsive differential equations with integral boundary conditions and one-dimensional p-Laplacian. Bound. Value Probl. 2011, Article ID 654871 (2011)

26. Cabada, A, Tersian, S: Existence and multiplicity of solutions to boundary value problems for fourth-order impulsive differential equations. Bound. Value Probl. 2014, 105 (2014)

27. Afrouzi, GA, Hadjian, A, Radulescu, VD: Variational approach to fourth-order impulsive differential equations with two control parameters. Results Math. 65, 371-384 (2014)

28. Sun, JT, Chen, HB, Yang, L: Variational methods to fourth-order impulsive differential equations. J. Appl. Math. Comput. 35, 323-340 (2011)

29. Xie, JL, Luo, ZG: Solutions to a boundary value problem of a fourth-order impulsive differential equation. Bound. Value Probl. 2013, 154 (2013)

30. Zhang, XM, Feng, MQ: Positive solutions for classes of multi-parameter fourth-order impulsive differential equations with one-dimensional singular p-Laplacian. Bound. Value Probl. 2014, 112 (2014)

31. Jankowski, T: Positive solutions of one-dimensional $p$-Laplacian boundary value problems for fourth-order differential equations with deviating arguments. J. Optim. Theory Appl. 149, 47-60 (2011)

32. Wang, HY: On the number of positive solutions of nonlinear systems. J. Math. Anal. Appl. 281, 287-306 (2003)

33. Guo, DJ, Lakshmikantham, V: Nonlinear Problems in Abstract Cones. Academic Press, New York (1988)

34. Jankowski, T: Positive solutions to third-order impulsive Sturm-Liouville boundary value problems with deviating arguments and one-dimensional p-Laplacian. Dyn. Syst. Appl. 20, 575-586 (2011)

\section{Submit your manuscript to a SpringerOpen ${ }^{\circ}$ journal and benefit from:}

- Convenient online submission

Rigorous peer review

- Immediate publication on acceptance

- Open access: articles freely available online

- High visibility within the field

- Retaining the copyright to your article 